\title{
Ultrafast layer-by-layer assembly of PDMS for boosting the H2 separation of a P84 membrane
}

\author{
Guozhen $\mathrm{Li}^{1}$, Zhihao $\mathrm{Si}^{1}$, Shuai Yang ${ }^{1}$, Tanlong Xue ${ }^{1}$, Jan Baeyens ${ }^{1}$, and Peiyong Qin ${ }^{1}$ \\ ${ }^{1}$ Beijing University of Chemical Technology
}

August 9, 2021

\begin{abstract}
BTDA-TDI/MDI (P84) has been widely applied in gas separation, however, pinholes or defects on P84 membrane surfaces were formed in the membrane preparation process and directly weaken its separation performance. Herein, we developed an ultrafast polydimethylsiloxane (PDMS) layer-by-layer assembly strategy via spraying method to heal the defects of the P84 membrane. Firstly, a very short assembly time 20-30 s was achieved by a UV-curing method. Secondly, both the PDMS spraying concentration and cycle times were optimized to strengthen the healing ability of PDMS. The P84-PDMS membrane with 3 wt \% PDMS spraying concentration and 2 spraying cycle times significantly improve the H2/CH4 selectivity from 62.99 to 231.92 with a satisfactory H2 permeance (20.85 GPU). The layer-by-layer PDMS assembly strategy for healing defects of P84 membrane displays outstanding comprehensive abilities, with an easy manufacturing based upon ultrafast curing and excellent gas separation performance based on the defect-free membrane structure.
\end{abstract}

\section{Hosted file}

Manuscript.docx available at https://authorea.com/users/429901/articles/533486-ultrafastlayer-by-layer-assembly-of-pdms-for-boosting-the-h2-separation-of-a-p84-membrane 